\title{
Quantum Entanglement Results from Quantum State Transition at Fast-Than-Light Speed with Matter Wave's Phase Velocity
}

\author{
Xinye Wang \\ Retired Scientist \\ Email: wang_xinye@yahoo.com
}

\begin{abstract}
Quantum entanglement is a primary feature of quantum mechanics and probably results from the quantum state's conservation and the quantum state's transition with the matter wave's phase velocity at the fast-than-light speed.

The quantum state transition of entangled particles proceeds with the phase velocity, while the observer measures the process with the electromagnetic or the light speed. This speed difference makes the causality law no longer fully valid except in certain areas.

\section{KEYWORDS}

Quantum Entanglement; Wave-Particle Duality; Quantum State Transition; Matter wave's Phase Transition; Causality Law
\end{abstract}

\section{INTRODUCTION}

Quantum entanglement is a primary feature of quantum mechanics and is defined to be one system whose quantum state cannot be described as individual but as an inseparable whole, even the particles are separated by a large distance. [1]

Measurements of physical properties on entangled particles such as position, momentum, spin and polarization can be found perfectly correlated. For example, if a pair of entangled particles is generated, their total spin is known to be zero, and one particle is found to have clockwise spin on an axis, then the spin of the other particle on the same axis, is found to be counterclockwise.

The counterintuitive performance of quantum mechanics that violates the local realism theory or causality law, were verified [2][3][4]. The measuring result at one point can be transmitted to a remote point very quickly, in some cases, the transmission speed can exceed over 10,000 times of the light speed [2][6].

The work summarized in this article is to explore the mechanism of quantum entanglement and deals with some problem about causality law. 


\section{QUANTUM ENTANGLEMENT OBEYS QUANTUM STATE'S CONSERVATION}

Quantum system can become entangled through various methods, the total momentum, angular momentum, energy, spin and so forth remains unchanged before and after the process. This process obeys conservations law.

As an example, a neutral $\pi$ meson with zero spin decays into an entangled pair of an electron and a positron, the total spin and electric charge keep unchanged before and after decay [5].

\section{QUANTUM STATES OF ENTANGLED PARTICLES TRANSIT WITH MATTER WAVE'S PHASE VELOCITY AT FAST-THAN-LIGHT SPEED}

According to the quantum mechanics theory, all particles from elementary particles to big molecules perform wave-particle duality property [7], the wave's property and the particle's property are equivalence, the wave's phase transition and the particle's state transition are closely related.

The speed of quantum state transition is faster than the light speed[2] [5] and the speed of matter wave's phase transmits at the phase velocity is also faster than the light speed (10), therefore, it can be supposed that the quantum state's transition proceeds with the matter wave's phase velocity.

The phase velocity is equal to the product of the frequency multiplied by the wavelength. If $\lambda$ is wavelength, $f$ is frequency, $\omega$ is angular frequency, $\hbar$ is reduced Planck constant, $E$ is energy, $P$ is momentum and $\omega=2 \pi f, k=2 \pi / \lambda$, the phase velocity $v_{p}$ is:

$v_{p}=\lambda f$

By the de Broglie hypothesis[8]:

$\mathrm{p}=\mathrm{h} / \lambda$

Formula (2) also can be written as

$p=\hbar k$

$E=\hbar \omega$

The phase velocity $v_{p}$ can be written as below:

$\mathrm{v}_{\mathrm{p}}=\lambda \mathrm{f}=\frac{\omega}{\mathrm{k}}=\frac{E / \mathrm{h}}{P / \mathrm{h}}=\frac{E}{\mathrm{P}}$

According to the special relativity, if $m$ is mass, $m$ 。 is rest (intrinsic) mass, $\gamma$ is Lorentz factor $\gamma=\frac{1}{\sqrt{1-\frac{V^{2}}{C^{2}}}}$

[9]:

$E=\gamma m 。 c^{2}$

$P=\gamma m 。 V$ 
$\mathrm{v}_{\mathrm{p}}=\frac{\mathbf{E}}{\mathbf{P}}=\frac{\mathrm{m} c^{2}}{\mathrm{mv}}=\frac{\gamma \mathrm{m} \circ c^{2}}{\gamma \mathrm{m} \circ \mathrm{v}}=\frac{c^{2}}{\mathrm{v}}$

In formula (8) v is particle's moving velocity i.e., the particle's group velocity and the value is always lower than the light speed in vacuum [10], so the particle's phase velocity, $v_{p}$ is always faster than the light speed in vacuum.

$\mathrm{V}<\mathrm{C}$

$\mathrm{v}_{\mathrm{p}}>\mathrm{C}$

The quantum entanglement's mechanism and cause can be regarded as the quantum state transition at the fast- than- light speed with the matter wave's phase velocity. Perhaps the so called "spooky action at a distance" (referred by Einstein) is exactly the matter wave's phase velocity.

\section{QUANTUM ENTANGLEMENT'S IMPACT ON CAUSALITY LAW}

According to causality law, events occur in chronological order. The event of cause happened before; the event of effect happens after [5].

Suppose that the entangled particle $\mathrm{Pa}$ and particle $\mathrm{Pb}$ are located at place $\mathrm{A}$ and place $\mathrm{B}$, the observer $O c$ is located at place $C$. Place $A, B, C$ join together to form a triangle, the distance between place $A$ and place $B$ is $S a b$, the distance between place $A$ and place $C$ is Sac, the distance between place $B$ and place $C$ is Sbc:

Let the time when the Pa's spin just turns to clockwise as the start point of time.

If the total spin of $\mathrm{Pa}$ and $\mathrm{Pb}$ is zero, when $\mathrm{Pa}$ 's spin turns to clockwise, $\mathrm{Pb}$ responds immediately with the phase velocity, $\mathrm{Vp}$ to turn its spin to counterclockwise.

At place $\mathrm{C}$, the observer Oc spends time Tc-ac

Tc-ac $=\frac{\text { sca }}{\boldsymbol{c}}$

to find Pa's spin just turns to clockwise and spends time Tc-bac

$\mathrm{Tc}-\mathrm{bac}=\frac{\boldsymbol{S a} \boldsymbol{b}}{\boldsymbol{V} \boldsymbol{p}}+\frac{S c b}{c}$

to find the $\mathrm{Pb}$ 's spin just turns to counterclockwise.

If Tc-bac $=\frac{S c b}{c}+\frac{S a b}{V p}>\mathrm{Tc}-\mathrm{ac}=\frac{S c \boldsymbol{a}}{\boldsymbol{c}}$, namely the observer Oc first to find the Pa's spin in clockwise and then to find $\mathrm{Pb}$ 's spin in counterclockwise i.e.

$\frac{S c b}{c}+\frac{S a b}{V p}>\frac{S c a}{c}$

$\frac{S c a-S c b}{C}<\frac{S a b}{V p}$

$\frac{S c a-S c b}{S a b}<\frac{C}{V p}$

As the Triangle law, $\quad \frac{S c a-S c b}{S a b}<1$ and $\frac{C}{V p}<1$, so

$\frac{S c a-S c b}{S a b}<\frac{C}{V p}<1$

When the case of (14) holds, the causality remains valid. 
As special cases, if $\mathrm{Scb}=\mathrm{Sca}$ or $\mathrm{Scb}>\mathrm{Sca}$, i.e. the observer Oc keeps the same distance from place $\mathrm{B}$ and place $A$ or the distance between observer Oc and place $B$ is longer than that between observer Oc and place $A$, it is clear from formula(14) that the causality is valid.

If Tc-bac $=\frac{S c b}{c}+\frac{S a b}{V p}<\mathrm{Tc}-\mathrm{ac}=\frac{S c a}{c}$, namely the observer Oc first to find $\mathrm{Pb}$ 's spin in counterclockwise and then to find the Pa's spin in clockwise i.e.

$\frac{S c a}{c}>\frac{S c b}{c}+\frac{S a b}{V p}$ i.e.

$\frac{S c a-S c b}{S a b}>\frac{C}{V p}$

As $\frac{S c a-S c b}{S a b}<1, \frac{C}{V p}<<1$ (in some case, $\frac{C}{V p}<0.0001$ ) and the value of $\frac{C}{V p}=\frac{P C}{E}(5)$ is independent of geometric position, the formula (15) is possible to be correct, depending on the values of Sca and Scb (only keeping the value $\mathrm{Sca}>\mathrm{Scb}$ ).

When formula (15) holds, the causality law is no longer valid.

In quantum entanglement system, if the quantum state transits with the speed that is faster than the observing or measuring speed, the causality law is no longer valid everywhere, except some areas where the formula (14) is satisfied, as special cases, the observer keeps the same distances from the place of cause event happened and the place of effect event happened or the distance between the observer and the place of effect event happened is longer than the distance between the observer and the place of cause event happened, the formula (14) must be true and the causality law remains valid.

\section{CONCLUSIONS}

The quantum entanglement probably results from the quantum state's transition with the matter wave's phase velocity that is faster than the light speed. The observing or measuring to the process in quantum entanglement system is made at the electromagnetic speed or the light speed,various counterintuitive phenomena in quantum entanglement arise from this speed difference.

The speed difference between the quantum state transition and measurement also makes the causality law in quantum entanglement system is no longer fully valid except in certain areas.

The author: Wang Xinye independent researcher, graduated from Tongji University, Shanghai, China in 1964 and now is a retired senior engineer (in China) and a retired scientist (in Singapore)

\section{REFERENCES}

[1] Laloe, Frank, Do We Really Understand Quantum Mechanics, Cambridge University Press,2012. 
[2] Yin, Juan; Cao, Yuan etc. "Bonding the speed of Spooky action at a distance". Physical Review Letters. 110(26):260407 .2013. https://doi:org/10.1103/PhysRevLett.110.260407.

[3] Matson,John (13 August 2012)."Quantum teleportation achieved over record distances",Nature News.https://doi:org/10.1038/nature.2012.11163.S2CID 124852641.

[4] Francis, Matthew. Quantum entanglement shows that reality can't be local Ars Technica,30 October 2012

[5] Griffiths, David J., Introduction to Quantum Mechanics (2 ${ }^{\text {nd }}$ ed), Prentice Hall,2004,ISBN 0-13-1118927

[6]Chinese Physicists Measure Speed of "Spooky Action at a Distance" Mit Technology Review. March 7. 2013

[7] French, Anthony: An Introduction to Quantum Physics, W.W. Norton Inc.; 1978.

[8] De Broglie, L Nobel. Lecture: The wave nature of the electron. The NobelFoundation;1929 (2008- 08-30)

[9] Yaakov Friedman, Physical Applications of Homogeneous Balls, Progress in Mathematical Physics; 2004.

[10] Wang Xinye, Information's Relativistic Convey with Matter Wave's Non-Dispersive Propagation, Physical Science International Journal 24(11),31 December 2020.

https://dol.org/10.36227/techrxiv.12987665.v1 